\title{
Gobernanza ambiental para ciudades del Sur Global: perspectivas teóricas
}

\author{
Environmental governance for cities in the Global South: theoretical perspectives \\ Governança ambiental para cidades do Sul Global: perspectivas teóricas
}

Recibido: 22/01/2021 | Revisado: 22/01/2021 | Acepto: 25/01/2021 | Publicado: 31/01/2021

\author{
Ignacio Loor \\ ORCID: https://orcid.org/0000-0003-4806-1032 \\ Universidad San Gregorio de Portoviejo, Ecuador \\ E-mail: iwloor@sangregorio.edu.ec \\ Lucía Rivadeneira \\ ORCID: https://orcid.org/0000-0001-5989-7703 \\ The University of Manchester, United Kingdom \\ E-mail: lucia.rivadeneirabarreiro@manchester.ac.uk \\ Julio Rivadeneira \\ ORCID: https://orcid.org/0000-0002-9494-7494 \\ Universidad Técnica de Manabí, Ecuador \\ E-mail: julio.rivadeneira@utm.edu.ec
}

\begin{abstract}
Resumen
La creciente urbanización informal plantea un desafío significativo a la gobernanza ambiental en el Sur Global. Este documento examina la literatura teórica que vincula la gobernanza ambiental con los asentamientos informales y la exclusión social en ciudades. Aunque el volumen de investigaciones sobre gobernanza ambiental está creciendo, la atención de los manuscritos existentes se centra en las ciudades del Norte Global, mientras que los conocimientos teóricos y los datos empíricos sobre las ciudades del Sur Global son aún incipientes. En una búsqueda de publicaciones de la última década en Scopus y Web of Science, sólo el 1\% de los manuscritos sobre gobernanza ambiental aborda los asentamientos informales. Los mecanismos de modernización ambiental y ecosocialismo se analizaron en cuanto a su idoneidad para abordar los desafíos ambientales en ciudades donde los asentamientos informales son prevalentes. Se desconoce la pertinencia de estos mecanismos y los marcos teóricos existentes para anticipar la capacidad de respuesta de la gobernanza urbana a las presiones ambientales en el Sur Global. Este documento crea conciencia de la necesidad de futuras investigaciones sobre gobernanza urbana donde los asentamientos informales son la norma. Esto es relevante para los académicos que trabajan en los Objetivos de Desarrollo Sostenible a escala de ciudad.
\end{abstract}

Palabras clave: Gobernanza ambiental; Asentamientos informales; Sur Global.

\begin{abstract}
Growing informal urbanization poses a significant challenge to environmental governance in the Global South. This paper examines the theoretical literature linking environmental governance to informal settlements and social exclusion in cities. Though the research output on environmental governance is growing, the attention is mainly focused on cities of the Global North, while theoretical insights and empirical data on cities of the Global South is still far from adequate. In a search of publications of the last decade in Scopus and Web of Science, only $1 \%$ of the research output on environmental governance addresses informal settlements. The mechanisms of environmental modernization and ecosocialism are considered for suitability to address environmental challenges where informal settlements are widespread. The relevance of these mechanisms and existing theoretical frameworks for anticipating urban governance responsiveness to environmental pressures in the Global South is unknown. The paper creates awareness of the need for future research on urban governance where informal settlements are the norm. This is relevant for scholars working on the Sustainable Development Goals at city scale.
\end{abstract}

Keywords: Environmental governance; Informal settlements; Global South.

\section{Resumo}

A crescente urbanização informal representa um desafio significativo para a governança ambiental no Sul Global. Este artigo examina a literatura teórica que liga a governança ambiental a assentamentos informais e exclusão social nas cidades. Embora a produção de pesquisa sobre governança ambiental esteja crescendo, a atenção está focada principalmente nas cidades do Norte Global, enquanto insights teóricos e dados empíricos sobre cidades do Sul Global ainda são incipientes. Em uma busca por publicações na última década em Scopus e Web of Science, apenas 1\% dos manuscritos de governança ambiental abordam assentamentos informais. Os mecanismos de modernização ambiental e ecosocialismo foram analisados quanto à sua adequação para enfrentar desafios ambientais em cidades onde assentamentos informais são predominantes. A relevância desses mecanismos e dos quadros teóricos existentes para antecipar a capacidade de resposta da governança urbana às pressões ambientais no Sul Global é desconhecida. Este artigo cria a conscientização sobre a necessidade de futuras pesquisas sobre governança urbana onde assentamentos 
informais são a norma. Isso é relevante para os estudiosos que trabalham nos Objetivos de Desenvolvimento Sustentável em escala de cidade.

Palavras-chave: Governança ambiental; Assentamentos informais; Sul Global.

\section{Introducción}

Este artículo examina la literatura teórica sobre gobernanza ambiental y su vínculo con el fenómeno de la urbanización informal y ofrece una base teórica a partir de la cual abordar desafíos socioambientales en ciudades del Sur Global ${ }^{1}$. Los efectos ambientales de la urbanización informal no son una preocupación reciente. La ex primera ministra india, Indira Gandhi, introdujo la noción de "la contaminación de la pobreza" en la Conferencia de Medio Ambiente de Estocolmo, en 1972. Ella reflexionó que no podemos mejorar el medio ambiente en un contexto apremiante de necesidades humanas insatisfechas. Desde Gandhi, la noción de contaminación de la pobreza ha inspirado una plétora de investigaciones multidisciplinarias sobre el vínculo pobreza - medio ambiente y sobre el desarrollo sostenible. Sin embargo, a pesar de los años y esfuerzos del multilateralismo por abordar los desafíos ambientales en el mundo, las ciudades del Sur Global siguen creciendo a un ritmo alarmante (Malakoff et al., 2016) y principalmente como asentamientos informales (Jain et al., 2015), lo que sugiere que sus políticas socioambientales han sido insuficientes.

Para dar una idea de la magnitud de este problema, según el Programa de las Naciones Unidas para los Asentamientos Humanos (UN, 2018), 4.200 millones de personas viven en ciudades hoy en día. Ello representa el 55 por ciento de la población mundial, dentro de la cual 900 millones viven en asentamientos informales. En las ciudades de América Latina, la prevalencia de residentes en asentamientos informales es en promedio del 46\% (UN, 2015). Varios estudiosos coinciden en que la creciente urbanización informal es una limitación clave para el desarrollo sostenible. Esto desafía el argumento de que el bienestar correlaciona positivamente con las emisiones de carbono (Druckman \& Birgitta, 2019; Givens, 2015; Jorgenson et al., 2010), lo que sugeriría que cuanto más pobre es un habitante, menos carbono emite. Sin embargo, la paradoja surge cuando la pobreza induce a las personas a adoptar prácticas contaminantes y emisoras de carbono para su supervivencia diaria. Esto es cotidiano en asentamientos informales, donde el acceso inadecuado a infraestructura básica como el agua, la electricidad, la movilidad y el saneamiento, fomenta la degradación del medio ambiente y el brote de enfermedades (Nzengya, 2018). Por lo tanto, se propone que una gobernanza ambiental efectiva en ciudades del Sur Global no puede prescindir de la participación activa de los residentes de los asentamientos informales.

McFarlane and Vasudevan (2014) se refieren a la informalidad como una serie de prácticas realizadas a través de medios no institucionalizados por aquellos que no pueden o no están dispuestos a pagar las alternativas oficialmente establecidas. Por lo tanto, las prácticas informales abarcan actividades y asociaciones que la legislación no prevé ni protege (Högenauer, 2014). Por ejemplo, el transporte urbano informal se refiere a los servicios de movilidad no regulados prestados a las personas con opciones de movilidad inadecuadas, generalmente los más pobres (Roy, 2009). El suministro informal de electricidad, de manera similar, ocurre en vecindarios sin medidores, a menudo alejados de las principales carreteras, a través de conexiones de cables clandestinas (Glover, 2011; Sengers \& Raven, 2014). Del mismo modo, el acceso al agua para los pobres urbanos a menudo depende de bombas manuales y pozos o camiones expendedores de agua (Bhattacharyya, 2014). Estas prácticas informales moldean sutilmente los procesos de transformación urbana (Coutard \& Rutherford, 2015; Roy, 2004, 2005), que a menudo son contaminantes y no suelen registrarse oportunamente (Elliott, 2013).

El resto del documento se organiza de la siguiente manera. La segunda parte presenta la metodología utilizada para llevar a cabo la revisión teórica de la literatura. La tercera parte presenta los resultados y la discusión organizados en cuatro

\footnotetext{
${ }^{1}$ La clasificación Norte Global y Sur Global se basa en el índice de desarrollo humano (HDI) del Programa de las Naciones Unidas para el Desarrollo (PNUD). El Norte Global lo conforman los 64 países que tienen un alto HDI (la mayoría de los cuales se sitúan al norte del paralelo 30 del norte), mientras que los 133 países restantes conforman al Sur Global.
} 
temas: la informalidad como aspecto de la exclusión social; exclusión socioespacial; desempeño de la gobernanza ambiental; y, mecanismos de gobernanza ambiental urbana. Por último, se proporcionan algunas conclusiones que ponen de relieve la necesidad de futuras investigaciones.

\section{Metodología}

El estudio tiene como objetivo identificar los fundamentos teóricos de la gobernanza ambiental y explorar su pertinencia en ciudades del Sur Global, donde los asentamientos informales son prevalentes. Se llevó a cabo una revisión teórica cualitativa de la literatura, lo que constituye un enfoque adecuado para examinar el cuerpo teórico en torno a un concepto (Labaree, 2013; Pereira et al., 2018). El estudio se propuso examinar las teorías existentes sobre gobernanza ambiental, el surgimiento de asentamientos informales y la relación entre ellos, para identificar posibles carencias teóricas para abordar la gobernanza ambiental en ciudades del Sur Global. Por consiguiente, se analizó y discutió distintas visiones sobre desafíos de gobernanza ambiental en varios contextos.

Se realizó una búsqueda de manuscritos en las bases de datos Scopus y Web of Science (WoS), utilizando las palabras clave gobernanza ambiental, asentamientos informales y exclusión social, sin límite de tiempo ni de categoría de manuscrito. Cuando el criterio de búsqueda es únicamente gobernanza ambiental, Scopus arroja 4,331 documentos y WoS 4,137. Un resultado preliminar interesante es que la producción científica sobre gobernanza ambiental ha crecido gradualmente a lo largo de la última década. $\mathrm{Al}$ agregar asentamientos informales en el criterio de búsqueda, las bases de datos produjeron tan solo 40 manuscritos cada una. Al combinar gobernanza ambiental con exclusión social, Scopus arrojó 124 manuscritos mientras WoS 96. Se realizó un análisis de contenido de los resúmenes de las dos últimas búsquedas, para identificar los temas y conceptos prominentes en torno a la gobernanza ambiental en ciudades del Sur Global. Luego de examinar los manuscritos filtrados, los resultados se organizaron en cuatro dimensiones que se presentan a continuación en la sección de resultados y discusión.

\section{Resultados y Discusión}

\subsection{La informalidad como tema de la exclusión social}

La informalidad se puede abordar como un producto de la exclusión social (Tókman, 2007), entendida como el fracaso de los marcos institucionales formales, ya sean democráticos o no, para incorporar las necesidades de todos los habitantes de un territorio. Cuando se consideran las vulnerabilidades ambientales de las ciudades, podemos suponer que la exclusión social afecta no sólo a los excluidos, sino también a los excluyentes y a todos los que habitan la ciudad. Las investigaciones sobre desarrollo sostenible (Srivastava \& Singh, 1996; Whitehead, 2013) y sobre ecología política urbana (Swyngedouw \& Heynen, 2003) han producido gran parte de lo que se conoce hoy sobre la relación entre la degradación ambiental y la exclusión social en las ciudades. Los estudiosos del desarrollo sostenible han encontrado una correlación positiva entre el crecimiento de las comunidades urbanas que concentran a los habitantes más pobres y el deterioro ecológico, que ha llegado a afectar a ciudades enteras y zonas rurales adyacentes (Cheng \& Zhang, 1999). Esto es relevante teniendo en cuenta que los espacios que concentran a los pobres urbanos crecen más rápido, en términos territoriales y demográficos, que los espacios que concentran mayor bienestar y oportunidades de prosperidad (Bolay, 2006).

Por otro lado, los ecologistas políticos urbanos se han enfocado en los cambios ecológicos resultantes de las políticas que fomentan el desarrollo económico desigual en las ciudades (Clark, 2020). En la última década, hay un creciente número de publicaciones académicas sobre justicia ambiental centrada en las vulnerabilidades de los asentamientos informales (Anguelovski et al., 2020; Reed \& George, 2011). No obstante, lo que no se ha discutido ampliamente en la literatura es cómo involucrar a los residentes de los asentamientos informales en la gobernanza ambiental de las ciudades. 
Comprender cómo la exclusión social deviene en prácticas informales para la supervivencia cotidiana puede proporcionar pistas para la gobernanza ambiental en ciudades del Sur Global. De hecho, se espera que las políticas que promueven inclusión social beneficien las prácticas y mecanismos de gobernanza ambiental (Silva, 2014). El término exclusión social surgió en el contexto político francés de la década de 1960, para referirse a personas aisladas del sistema de seguridad social (Lenoir, 1974). Estos comprendían personas mutiladas, ancianos, infantes maltratados, personas con problemas mentales, con movilidad reducida, drogadictos y suicidas, por mencionar algunos. Pero el alcance en el uso y significado de exclusión social se fue ampliando gradualmente. A finales de la década de 1980, el término también se usó para referirse a los migrantes víctimas de xenofobia en Europa occidental (Lepianka, 2003). Posteriormente, en los debates sobre política social de los Estados Unidos en la década de 1990, la exclusión social se convirtió en un concepto clave para explicar las causas de la pobreza y la desigualdad (Atkinson \& Da Voudi, 2000). La noción de "excluido socialmente" reemplazó al término peyorativo "subclase" que había sido convencional en el discurso político. Gunnar (1963) había introducido el concepto de subclase para referirse a las personas sin empleo y con actitudes apáticas sobre los logros de la nación.

Así, el concepto de exclusión social evolucionó a partir de la fusión de la interpretación francesa de aislamiento social, las preocupaciones anglosajonas sobre las desigualdades de ingresos y la caracterización norteamericana de la subclase. Más recientemente, la exclusión social también se convirtió en un marco teórico a partir del cual comprender las causas de la pobreza en sus múltiples dimensiones, incluida la de las privaciones ecosistémicas y la justicia ambiental (Pornet et al., 2012; Wolch et al., 2014).

En cuanto a las causas de la exclusión social, Silver (1994) ha distinguido tres paradigmas: la noción francesa de solidaridad social, que atribuye la exclusión social a los fracasos institucionales de los sistemas políticos que conectan a las personas con la sociedad; la visión angloamericana de la especialización, que concibe el orden social como redes de intercambios voluntarios dentro de comunidades de practicantes, en cuyo caso, la exclusión se considera voluntaria; y, el paradigma de monopolio de Europa Occidental, donde los poderes políticos privilegian los intereses de los ricos sobre los pobres. Desde estas perspectivas, la inclusión o exclusión es una cuestión de la relación de las personas con la sociedad en general, que da forma tanto al bienestar como a la pobreza.

La exclusión social se trata de las relaciones entre los excluidos y los incluidos, o el individuo excluido y la sociedad. Su comprensión permite discusiones sobre los actores e instituciones que promueven y restringen la producción de relaciones constructivas (Room, 1995). Tomando la reciprocidad como característica intrínseca de las relaciones humanas, la exclusión social es la incapacidad de dar, y en consecuencia, de recibir (Alcock, 2006). Desde una perspectiva económica, la exclusión social refleja como la relación de un individuo con la sociedad le permite sufragar o no sus necesidades cotidianas (Giraud et al., 2013). Esto incluye sufragar el acceso a la infraestructura básica y a un entorno seguro. En otras palabras, la pobreza es una privación de capacidades en lugar de, simplemente, un ingreso inadecuado. El argumento relacional sugiere que, más allá del desempleo y las carencias materiales, la pobreza se define en la capacidad de un individuo para involucrarse en relaciones recíprocas con actores e instituciones de la sociedad en general. Esta visión de la pobreza como una cuestión relacional puede informar a las políticas para abordar sus múltiples dimensiones (Haan \& Maxwell, 1998; Lepianka, 2003), incluyendo las causas de los problemas ambientales de la ciudad.

Por citar un ejemplo, considérese las grandes ciudades latinoamericanas, donde la concentración espacial de la pobreza a menudo correlaciona con la violencia (Berkman, 2007). Moser \& McIlwaine (2000) llevaron a cabo investigaciones en ciudades de Guatemala y Colombia utilizando el marco de exclusión social. Una restricción prominente para que los residentes de los asentamientos informales se relacionen con el resto de la sociedad es el estigma y la reputación de vivir en lugares considerados peligrosos. Por lo general, esto fomenta la confrontación sistémica entre los residentes de estas comunidades con la policía. En los asentamientos informales (favelas) de Río de Janeiro, Brasil, la policía y el ejército han unido fuerzas para 
enfrentar a redes de narcotráfico que involucran a jóvenes residentes. Esto ha provocado una situación de desconfianza y miedo entre los residentes, y entre los residentes y la policía (Roy, 2005). La desconfianza exacerba la incapacidad de relacionarse productivamente con otros actores e instituciones de la sociedad (Goshin et al., 2014) y, por lo tanto, restringe la capacidad de incluir a los residentes de los asentamientos informales en los esquemas de gobernanza ambiental.

\subsection{Exclusión socioespacial}

Los pobres y socialmente excluidos de las ciudades se suelen concentrar espacialmente. En países del Sur Global, es normal que este fenómeno se materialice como asentamientos informales. Por lo general, estos espacios se erigen en localidades remotas e inhóspitas, con restricciones para desplazarse a las áreas centrales de las ciudades, y la economía de sus habitantes es en su mayoría informal. Además, sus casas son a menudo precarias, lo que exacerba los riesgos a la salud (Cass et al., 2005; Lupton \& Power, 2002). Con frecuencia, habitan en ecosistemas degradados con acceso a servicios e instalaciones públicas inadecuado (Oberwittler, 2007). Además, los residentes de asentamientos informales a menudo participan en redes de trabajadores informales que se desarrollan lejos de los vínculos con la educación, la capacitación y el empleo formal. Esto refleja la investigación de Lupton \& Power (2002) en el sentido de que la concentración espacial de personas desempleadas limita las redes de búsqueda de empleo.

Lefebvre y Nicholson-Smith (2011) introdujeron la noción de espacio social como un facilitador material de la innovación y la producción, que resulta de la interacción de los objetos naturales a la mano con las relaciones sociales. Las interacciones en los espacios de los excluidos producen desventaja (Kabeer, 2000), lo que exacerba la condición de la pobreza y a menudo la hace crónica. Desde esta perspectiva, abordar la pobreza implica fomentar el desarrollo de los espacios sociales donde se concentran los pobres. Esto es clave para diseñar esquemas de gobernanza ambiental en las ciudades del Sur Global, ya que la mera intervención ambiental en asentamientos informales, sin que ello implique mejoras en las condiciones de vida de sus habitantes, podría caer en lo que se conoce como estetización de la pobreza (Roy \& Crane, 2015).

Sin embargo, esta forma de pensar es a menudo objeto de controversia. Si las políticas deben abordar el espacio de los socialmente excluidos o privilegiar a los sectores económicos que demandan mano de obra de los pobres es un debate inconcluso (Andersson \& Musterd, 2005). A menudo, lo primero implica inversiones en infraestructura básica y social ${ }^{2}$ en espacios donde se disputa la propiedad de la tierra. Los recursos financieros para esas inversiones frecuentemente dependen de ingresos fiscales. Lo segundo, por otro lado, busca estimular el consumo privado mediante incentivos fiscales, y con ello estimular la demanda de empleo. No obstante, este mecanismo puede reducir la capacidad financiera de los gobiernos para invertir en infraestructura social. Para Lupton (2003) las políticas aisladas difícilmente pueden ofrecer soluciones sostenibles a la exclusión socioespacial, por lo que la decisión sobre si las políticas deben intervenir el espacio o estimular la demanda laboral no debería constituir un dilema.

\subsection{Desempeño de la gobernanza ambiental}

La gobernanza ambiental en ciudades donde la exclusión socioespacial es común debe considerar las prácticas cotidianas de los excluidos. La gobernanza comprende los instrumentos y mecanismos disponibles para dirigir una sociedad en una dirección deseada (Lafferty, 2006; Jon Pierre, 2000). La gobernanza es un aspecto crítico del desarrollo sostenible. El Objetivo de Desarrollo Sostenible (ODS) 17 fomenta las asociaciones entre el sector público, el sector privado y la sociedad civil para abordar el desarrollo sostenible en todas sus escalas. La producción de gobernanza implica que las redes de

\footnotetext{
${ }^{2} \mathrm{La}$ infraestructura social se refiere a las instalaciones que sientan las bases de la vida pública, como bibliotecas, escuelas, jardines de infancia y hospitales.
} 
instituciones y comunidades gobernadas de manera autónoma construyan acuerdos para ejercer poder sobre los desafíos de interés colectivo (Joseph, 1999; Kjær, 2004; Rhodes, 1997). Por lo tanto, la gobernanza para el desarrollo sostenible exige acuerdos y compromisos sobre los desafíos ambientales, sociales y económicos prioritarios.

Anticipar el desempeño de una configuración de gobernanza, en términos de capacidad para abordar los desafíos económicos, sociales y ambientales de la ciudad, es una tarea esencial para el proceso de desarrollo sostenible. En la literatura sobre estudios urbanos, dos marcos prevalentes para el análisis de la gobernanza son la teoría de régimen urbano (Mossberger, 2009) y la teoría de gobernanza urbana (Giersig, 2008; Jon Pierre, 2014). Si bien ambos enfoques reconocen que gobernar las presiones ambientales de una ciudad exige significativamente más recursos de los que normalmente maneja un gobierno municipal (Libertun de Duren \& Guerrero Compeán, 2015; Stoker, 2000), existen diferencias en la forma en que se conciben los actores involucrados.

La teoría de régimen urbano surgió en los Estados Unidos desde la publicación de Stone (1989) sobre la ciudad de Atlanta. Un régimen es una coalición transitoria de gobierno entre las autoridades y actores corporativos. La distribución de poder entre los actores de la coalición da forma a las características del desarrollo (Kilburn, 2004) y la transformación del medio ambiente. La validez de este marco ha sido objeto de críticas debido a su énfasis en el localismo, puesto que se enfoca en cómo surgen los regímenes mientras desconoce el poder influyente de los actores no corporativos y agentes externos en la formulación de políticas (Jones, 1998; Mossberger, 2009; Pierre, 2014).

Por otro lado, la teoría de la gobernanza urbana es dominante en las discusiones sobre la política urbana de Europa Occidental (Pierre, 2014). El liderazgo de la ciudad involucra a actores que no sólo representan al sector corporativo, sino también a una gama de intereses como la prestación de servicios, el cambio climático, la inmigración y otros. Según Pierre (1999), este enfoque es coherente con nuevos modos de gobernanza, los que suponen una forma menos jerárquica y más colaborativa y deliberativa de participación entre los actores públicos y privados. Este enfoque es cada vez más frecuente en debates sobre política ambiental urbana en Europa (Bäckstrand et al., 2010).

Hay dos diferencias clave en estas teorías que merece la pena destacar: en primer lugar, el ciclo de vida de los actores involucrados en la formulación de políticas. Mientras los regímenes son coaliciones transitorias, el paradigma de la gobernanza urbana considera que las redes y asociaciones involucradas suelen ser estables. $\mathrm{Y}$ en segundo lugar, la dimensión central del desarrollo (Barca et al., 2012). La visión del régimen privilegia los aspectos económicos de las políticas de desarrollo (Jessop, 1997), probablemente porque el capital privado es un socio natural de los regímenes. En cambio, cuando las ideas de desarrollo priorizan la inclusión social y la cohesión territorial (Barca et al., 2012), el marco de gobernanza urbana parece más útil para anticipar posibles desempeños en lo ambiental. Sin embargo, si bien la perspectiva de gobernanza urbana hace referencia a la inclusión social, esto no necesariamente implica a los asentamientos informales. Por lo tanto, no constituye un marco teórico ampliamente representativo de los desafíos ambientales del Sur Global. La prevalencia del urbanismo informal en el Sur Global plantea desafíos teóricos que guíen la configuración de una gobernanza ambiental efectiva.

\subsection{Mecanismos de gobernanza ambiental urbana}

Una de las principales preocupaciones de la gobernanza ambiental es la capacidad para controlar la movilidad de la contaminación (Bäckstrand et al., 2010). La contaminación no es un ente estático, sino que se desplaza por medio de la atmósfera, la litósfera y la hidrósfera. En ciudades del Sur Global, esto implica que los desafíos ambientales en los asentamientos informales afectan a los residentes del espacio formal de la ciudad y viceversa. Pepper (1998) examinó los mecanismos para abordar estas preocupaciones ambientales desde las perspectivas de la modernización ecológica y el ecosocialismo. Estos dos paradigmas abordan la movilidad de la contaminación de diferentes maneras y reflejan el dilema entre invertir en infraestructura básica y 
social en el espacio de los socialmente excluidos o estimular el consumo mediante la reducción de impuestos para abordar la exclusión social mediante la creación de empleo de los sectores más empobrecidos, como se discutió anteriormente.

La noción de modernización ecológica surgió como discurso político en la década de 1980 en Europa occidental para armonizar el desarrollo económico y social con la preservación del medio ambiente. Como discurso político, esta perspectiva es funcional para los regímenes urbanos y para políticas que privilegian a los sectores económicos que utilizarían la oferta laboral de los pobres por encima de la inversión en infraestructura. El objetivo, "en pocas palabras, es lograr resultados ambientales positivos a través de mecanismos de mercado" (Bakker, 2014 p.475). Las políticas derivadas de esta perspectiva han sido sujeto de crítica por el énfasis en los intereses comerciales (Cohen, 1999; Newell, 2008) y por promover políticas de uso de suelo que han causado desplazamiento de personas vulnerables (Cabello, 2008). Además, Copeland (2010) ha criticado que, si bien la modernización ecológica puede restaurar la calidad ambiental en las ciudades, esto se logra por un mecanismo de reubicación de la producción de contaminación en lugar de la eliminación de esta. La modernización ecológica, a menudo denominada ecologismo de libre mercado, implica incentivos basados en el mercado en lugar de regulaciones para reducir la contaminación (Christoff, 1996). Algunos ejemplos de estos mecanismos guardan relación con la mercantilización del suelo, las certificaciones ecológicas y el comercio de emisiones de carbono, que funcionan con mínima participación gubernamental (Backstrand, 2010).

En cambio, la noción de ecosocialismo se basa en la crítica marxista del capitalismo como un paradigma destructivo. Desde esta perspectiva, la sociedad tiene como objetivo "la racionalidad ecológica, el control democrático, la igualdad social y el predominio del valor de uso sobre el valor de intercambio" (Löwy, 2007 p.1). Para los ecosocialistas, la organización social capitalista ha producido desigualdades socioecológicas en ciudades de todo el mundo. En cuanto a la gobernanza, el ecosocialismo propone el fortalecimiento de instituciones guardianas del estado de bienestar. Esto refleja las opiniones de los teóricos de la gobernanza urbana. No obstante, los ecosocialistas reconocen las limitaciones de las políticas fiscales, por lo que sugieren mecanismos alternativos como cambios en los sistemas de transporte que promuevan la movilidad activa y minimizan la dependencia de la movilidad motorizada, racionalización en el consumo de energía, políticas de vivienda social y la creación de empleos verdes (Singh \& Bhusal, 2014). Estos mecanismos parecen converger con el discurso sobre políticas para abordar la exclusión social. No obstante, es necesario explorar estos mecanismos empíricamente en ciudades del Sur Global antes de que se pueda llegar a una conclusión sobre su practicidad para la gobernanza ambiental donde los asentamientos informales son la norma.

\section{Conclusión}

En este documento se ha examinado la literatura teórica sobre gobernanza ambiental y su relación con los asentamientos informales y la exclusión social en ciudades del Sur Global. Si bien es notable que el interés científico en la gobernanza ambiental ha incrementado durante la última década, la pertinencia de estos trabajos para abordar desafíos de ciudades con evidente desigualdad en el acceso a bienes y servicios básicos y presencia de asentamientos informales es aún discutible. En consecuencia, la gobernanza ambiental es un concepto desarrollado principalmente en el contexto del Norte Global, mientras que persiste una escasez de investigación sobre el tema en el Sur Global. Del mismo modo, las teorías existentes sobre régimen y gobernanza urbanos, así como los mecanismos de modernización ecológica y ecosocialismo han sido poco explorados desde la perspectiva de los desafíos ambientales de las ciudades del Sur Global.

En consecuencia, urge promover el desarrollo de nuevos marcos teóricos que guíen la investigación empírica pertinente a los que hoy son los espacios de mayor crecimiento urbano del mundo en términos espaciales y demográficos. Para futuras investigaciones es necesario examinar cómo las organizaciones comunitarias que surgen en asentamientos informales se relacionan con su entorno natural y qué les incentiva a involucrarse en trabajos que impliquen remediación y preservación ambiental. 


\section{Referencias}

Alcock, P. (2006). Understanding poverty (3rd ed). Palgrave Macmillan.

Andersson R., \& Musterd, S. (2005). Area-based policies: A critical appraisal. Tijdschrift voor economische en sociale geografie, 96(4), 377-389. https://doi.org/10.1111/j.1467-9663.2005.00470.x

Anguelovski, I., Brand, A. L., Connolly, J. J. T., Corbera, E., Kotsila, P., Steil, J., Garcia-Lamarca, M., Triguero-Mas, M., Cole, H., Baró, F., Langemeyer, J., del Pulgar, C. P., Shokry, G., Sekulova, F., \& Argüelles Ramos, L. (2020). Expanding the Boundaries of Justice in Urban Greening Scholarship: Toward an Emancipatory, Antisubordination, Intersectional, and Relational Approach. Annals of the American Association of Geographers, 110(6), 1743-1769. https://doi.org/10.1080/24694452.2020.1740579

Atkinson, R., \& Da Voudi, S. (2000). The Concept of Social Exclusion in the European Union: Context, Development and Possibilities. JCMS: Journal of Common Market Studies, 38(3), 427-448. https://doi.org/10.1111/1468-5965.00229

Bäckstrand, K., Kahn, J., Kronsell, A., \& Lövbrand, E. (2010). Environmental Politics and Deliberative Democracy. Edward Elgar Publishing. https://doi.org/10.4337/9781849806411

Bakker, K. (2014). The Business of Water: Market Environmentalism in the Water Sector. Annual Review of Environment and Resources, 39(1), 469-494. https://doi.org/10.1146/annurev-environ-070312-132730

Barca, F., McCann, P., \& Rodríguez-Pose, A. (2012). The case for regional development intervention: place-based versus place-neutral approaches. Journal of Regional Science, 52(1), 134-152. https://doi.org/10.1111/j.1467-9787.2011.00756.x

Berkman, H. (2007). Social exclusion and violence in Latin America and the Caribbean.

Bhattacharyya, S. C. (2014). Business issues for mini-grid-based electrification in developing countries. En Mini-Grids for Rural Electrification of Developing Countries (pp. 145-164). Springer.

Bolay, J.-C. (2006). Slums and Urban Development: Questions on Society and Globalisation. The European Journal of Development Research, 18(2), 284-298. https://doi.org/10.1080/09578810600709492

Cabello, J. (2008). The Politics of the Clean Development Mechanism: A ton of carbon here is the same as a ton of carbon there? Institute of Social Studies.

Cass, N., Shove, E., \& Urry, J. (2005). Social exclusion, mobility and access. The sociological review, 53(3), 539-555. https://doi.org/10.1111/j.1467954X.2005.00565.X

Cheng, J. Y., \& Zhang, M. (1999). Regional differences and sustainable development in China. Journal of Chinese Political Science, 5(1), 1-50. https://doi.org/10.1007/BF02876851

Christoff, P. (1996). Ecological modernisation, ecological modernities. Environmental Politics, 5(3), 476-500. https://doi.org/10.1080/09644019608414283

Clark, A. (2020). Interrogating Resilience: Governing Disasters in Metro Manila Post-Typhoon Ondoy. https://qspace.library.queensu.ca/handle/1974/28221

Cohen, M. J. (1999). Sustainable Development and Ecological Modernisation: National Capacity for Rigorous Environmental Reform. En D. RequierDesjardins, C. Spash, \& J. van der Straaten (Eds.), Environmental Policy and Societal Aims, 2, 103-128. Springer Netherlands. https://doi.org/10.1007/978-94011-4521-3_5

Copeland, B. (2010). The pollution heaven hypothesis. En K. Gallagher (Ed.), Handbook on Trade and the Environment. (pp. 60-70). Edward Elgar Publishing.

Coutard, O., \& Rutherford, J. (2015). Beyond the Networked City: Infrastructure reconfigurations and urban change in the North and South. Taylor \& Francis. https://books.google.co.uk/books?id=KsIOCwAAQBAJ

Druckman Angela \& Gatersleben Birgitta. (2019). A time-use approach: High subjective wellbeing, low carbon leisure. Journal of Public Mental Health, 18(2), 85-93. https://doi.org/10.1108/JPMH-04-2018-0024

Elliott, J. A. (2013). An introduction to sustainable development (Fourth Edition). Routledge, Taylor \& Francis Group.

Giersig, N. (2008). Multilevel Urban Governance and the "European City”: Discussing metropolitan reforms in Stockholm and Helsinki. Springer Fachmedien. https://public.ebookcentral.proquest.com/choice/publicfullrecord.aspx?p=748729

Giraud, G., Renouard, C., l'Huillier, H., de la Martinière, R., \& Sutter, C. (2013). Relational Capability: A Multidimensional Approach. ESSEC Business School. https://hal-essec.archives-ouvertes.fr/hal-00815586

Givens, J. E. (2015). Urbanization, slums, and the carbon intensity of well-being: Implications for sustainable development. Human Ecology Review, 22(1), $107-128$.

Glover, L. (2011). Public transport as a common pool resource.

Goshin, L. S., Byrne, M. W., \& Henninger, A. M. (2014). Recidivism after Release from a Prison Nursery Program. Public Health Nursing, 31(2), 109-117. https://doi.org/10.1111/phn.12072

Gunnar, M. (1963). Challenge to Affluence. Random House.

Haan, A. de, \& Maxwell, S. (1998). Editorial: Poverty and Social Exclusion in North and South. IDS Bulletin, 29(1), 1-9. https://doi.org/10.1111/j.17595436.1998.mp29001001.x 
Högenauer, A.-L. (2014). Formal rules and informal cooperation: Inter-governmental relations in domestic european policy making in comparative perspective. Regional \& Federal Studies, 24(3), 321-340.

Jain, M., Knieling, J., \& Taubenböck, H. (2015). Urban transformation in the National Capital Territory of Delhi, India: The emergence and growth of slums? Habitat International, 48, 87-96. https://doi.org/10.1016/j.habitatint.2015.03.020

Jessop, B. (1997). A Neo-Gramscian Approach to the Regulation of Urban Regimes: Accumulation Strategies, Hegemonic Projects, and Governance. En M. Lauria, Reconstructing Urban Regime Theory: Regulating Urban Politics in a Global Economy (pp. 51-74). SAGE Publications, Inc. https://doi.org/10.4135/9781483327808.n4

Jones, M. (1998). Restructuring the local state: Economic governance or social regulation? Political Geography, 17(8), 959-988. https://doi.org/10.1016/S09626298(97)00090-5

Jorgenson, A. K., Rice, J., \& Clark, B. (2010). Cities, Slums, and Energy Consumption in Less Developed Countries, 1990 to 2005. Organization \& Environment, 23(2), 189-204. https://doi.org/10.1177/1086026610368376

Joseph, R. A. (Ed.). (1999). State, conflict, and democracy in Africa. L. Rienner.

Kabeer, N. (2000). Social Exclusion, Poverty and Discrimination Towards an Analytical Framework. IDS Bulletin, 31(4), 83-97. https://doi.org/10.1111/j.17595436.2000.mp31004009.x

Kilburn, H. W. (2004). Explaining U.S. Urban Regimes: A Qualitative Comparative Analysis. Urban Affairs Review, 39(5), 633-651. https://doi.org/10.1177/1078087403262861

Kjær, A. M. (2004). Governance. Polity Press.

Labaree, R. (2013). LibGuides. Organizing Your Social Sciences Research Paper. Writing a Field Report. https://libguides.usc.edu/writingguide

Lafferty, W. M. (2006). Governance for sustainable development: The challenge of adapting form to function. Edward Elgar.

Lefebvre, H., \& Nicholson-Smith, D. (2011). The production of space (Nachdr.). Blackwell.

Lenoir, R. (1974). Les Exclus: Un Francais Sur Dix Le Seuil.

Lepianka, D. (2003). What Is Social Exclusion—A Brief Introduction to the Concept. The Polish Foreign Affairs Digest, 1(6), 85-109.

Libertun de Duren, N., \& Guerrero Compeán, R. (2015). Growing Resources for Growing Cities. Density and the Cost of Municipal Public Services in Brazil, Chile, Ecuador, and Mexico. (IDB WORKING PAPER SERIES Nº IDB-WP-634).

Löwy, M. (2007). Eco-socialism and democratic planning. Socialist Register, 43. (Vol. 43). Socialist Register.

Lupton, R. (2003). Neighbourhood effects': Can we measure them and does it matter? (73) [CASEpaper]. Centre for Analysis of Social Exclusion, London School of Economics and Political Science. http://sticerd.lse.ac.uk/case

Lupton, R., \& Power, A. (2002). Social exclusion and neighbourhoods. En Understanding Social Exclusion (pp. 118-140). Oxford University Press.

Malakoff, D., Wigginton, N., Fahrenkamp-Uppenbrink, J., \& Wible, B. (2016). Rise of the urban planet. Science. https://doi.org/doi:10.1126/science.aaf5729

McFarlane, C., \& Vasudevan, A. (2014). Informal infrastructures. Adey P, Bissell D, Hannam K, Merriman P \& Sheller M. Handbook of Mobilities, Routledge, $256-264$

Moser, C. O. (2001). La violencia en el contexto del posconflicto, según la percepción de comunidades urbanas pobres de Guatemala. Región de Latinoamérica y el Caribe, Banco Mundial.

Moser, C. O., \& Mcllwaine, C. (2000). La violencia y la exclusión en Colombia según la percepción de comunidades urbanas pobres. Región de Latinoamérica y el Caribe, Banco Mundial.

Mossberger, K. (2009). Urban Regime Analysis. En J. Davies \& D. Imbroscio, Theories of Urban Politics (pp. 40-54). SAGE Publications Ltd. https://doi.org/10.4135/9781446279298.n4

Newell, P. (2008). The political economy of global environmental governance. Review of International Studies, 34(3), 507-529. Cambridge Core. https://doi.org/10.1017/S0260210508008140

Nzengya, D. M. (2018). Improving water service to the urban poor through delegated management: Lessons from the city of Kisumu, Kenya. Development Policy Review, 36(2), 190-202. https://doi.org/10.1111/dpr.12361

Oberwittler, D. (2007). The Effects of Neighbourhood Poverty on Adolescent Problem Behaviours: A Multi-level Analysis Differentiated by Gender and Ethnicity. Housing Studies, 22(5), 781-803. https://doi.org/10.1080/02673030701474727

Pepper, D. (1998). Sustainable development and ecological modernization: A radical homocentric perspective. Sustainable Development, 6(1), 1-7. https://doi.org/10.1002/(SICI)1099-1719(199803)6:1<1::AID-SD83>3.0.CO;2-8

Pereira, A. S., Shitsuka, D. M., Parreira, F. J., \& Shitsuka, R. (2018). Metodologia da investigação científica. UAB / NTE / UFSM. https://repositorio.ufsm.br/bitstream/handle/1/15824/Lic_Computacao_Metodologia-Pesquisa-Cientifica.pdf?sequence=1. 
Research, Society and Development, v. 10, n. 1, e56410112278, 2021

(CC BY 4.0) | ISSN 2525-3409 | DOI: http://dx.doi.org/10.33448/rsd-v10i1.12278

Pierre, Jon. (1999). Models of Urban Governance: The Institutional Dimension of Urban Politics. Urban Affairs Review, 34(3), 372-396. https://doi.org/10.1177/10780879922183988

Pierre, Jon. (2000). Debating governance: Authority, steering, and democracy. OUP Oxford.

Pierre, Jon. (2014). Can Urban Regimes Travel in Time and Space? Urban Regime Theory, Urban Governance Theory, and Comparative Urban Politics. Urban Affairs Review, 50(6), 864-889. https://doi.org/10.1177/1078087413518175

Pornet, C., Delpierre, C., Dejardin, O., Grosclaude, P., Launay, L., Guittet, L., Lang, T., \& Launoy, G. (2012). Construction of an adaptable European transnational ecological deprivation index: The French version. Journal of epidemiology and community health, 6(11), 982-989. https://doi.org/10.1136/jech2011-200311

Reed, M. G., \& George, C. (2011). Where in the world is environmental justice? Progress in Human Geography, 35(6), 835-842. https://doi.org/10.1177/0309132510388384

Rhodes, R. A. W. (1997). Understanding governance: Policy networks, governance, reflexivity, and accountability. Open University Press.

Room, G. (1995). Beyond the threshold: The measurement and analysis of social exclusion. Policy Press.

Roy, A. (2004). Transnational trespassings: The geopolitics of urban informality. Urban Informality: Transnational Perspectives from the Middle East, Latin America, and South Asia, 289-317.

Roy, A. (2005). Urban Informality: Toward an Epistemology of Planning. Journal of the American Planning Association, 71(2), 147-158. https://doi.org/10.1080/01944360508976689

Roy, A. (2009). Why India cannot plan its cities: Informality, insurgence and the idiom of urbanization. Planning theory, 8(1), 76-87.

Roy, A., \& Crane, E. S. (Eds.). (2015). Territories of poverty: Rethinking North and South. University of Georgia Press.

Sengers, F., \& Raven, R. (2014). Metering motorbike mobility: Informal transport in transition? Technology Analysis \& Strategic Management, $26(4), 453-468$.

Silva, E. (2014). Environment and Citizenship in Latin America: Natures, Subjects, and Struggles, edited by Alex Latta and Hannah Wittman. CEDLA Latin American Studies 101. New York: Berghahn Books. 2012. Pp. Xii+254. £43 (hb). ISBN 978-0-85745-747-9. Journal of Agrarian Change, 14(3), 475-477. https://doi.org/10.1111/joac.12073

Silver, H. (1994). Social Exclusion and Social Solidarity: Three Paradigms. International Labour Review, 133(5-6), 531-578.

Singh, P., \& Bhusal, L. (2014). Austerity, Welfare State and Eco-Socialism. Economic \& Political Week, 49(39).

Srivastava, A., \& Singh, R. C. (1996). Slums and associated problems: A case study of Bhilai, an industrial city, India. International Journal of Environmental Studies, 50(1), 51-60. https://doi.org/10.1080/00207239608711038

Stoker, G. (2000). Urban Political Science and the Challenge of Urban Governance. En J Pierre (Ed.), Debating Governance: Authority, Steering, and Democracy. Oxford University Press. http://eprints.soton.ac.uk/id/eprint/47311

Stone, C. N. (1989). Regime politics: Governing Atlanta, 1946-1988. University Press of Kansas.

Swyngedouw, E., \& Heynen, N. C. (2003). Urban Political Ecology, Justice and the Politics of Scale. Antipode, 35(5), 898-918. https://doi.org/10.1111/j.14678330.2003.00364.x

Tókman, V. (2007). Informalidad, inseguridad y cohesión social en América Latina. CEPAL. https://repositorio.cepal.org/handle/11362/6135

UN. (2015). Indicator 7.10 Proportion of urban population living in slums. Recuperado el 1 de septiembre de 2020, de Millennium Development Goals Indicators website: http://mdgs.un.org

UN. (2018, mayo 16). $68 \%$ of the world population projected to live in urban areas by 2050 says UN. www.un.org. https://bit.ly/32yNj5D

Whitehead, M. (2013). Sustainability. En C. Death (Ed.), Critical Environmental Politics (p. 257).

Wolch, J. R., Byrne, J., \& Newell, J. P. (2014). Urban green space, public health, and environmental justice: The challenge of making cities 'just green enough'. Landscape and Urban Planning, 125, 234-244. 